\title{
Optimization of canine interleukin-12 production using a baculovirus insect cell expression system
}

\author{
Cristiane Garboggini Melo de Pinheiro ${ }^{1,2^{*}}$, Mayara de Oliveira Pedrosa', Naiara Carvalho Teixeira', \\ Ana Paula Dinis Ano Bom ${ }^{3}$, Monique M. van Oers ${ }^{4}$ and Geraldo Gileno de Sá Oliveira ${ }^{1,5^{*}}$
}

\begin{abstract}
Background: Interleukin-12 is an important cytokine in mediating cellular immune responses.

Results: Recombinant single-chain canine IL-12 was produced in a baculovirus-insect cell system with the aim of conducting further studies on modulation of immune responses in dogs. To optimize the production of recombinant canine IL-12, a classical baculovirus and a modified vector (chitinase A and v-cathepsin knockout) were used containing a native or an optimized insert of canine IL-12. The optimized IL-12 construct contained the GP64 signal peptide and was synthesized with optimized codons for expression in Trichoplusia ni cells. Dot-blot and Western blot analysis showed the highest production levels of recombinant IL-12 protein by the use of the modified baculovirus vector containing the optimized insert, at a multiplicity of infection of five and at $48 \mathrm{~h}$ after infection. The recombinant cytokine was successfully purified and showed a good degree of purity, integrity, folding, and yield, with very little endotoxin contamination. Recombinant canine IL-12 induced IFN- $\gamma$ in canine lymphocytes, indicating that it was biologically active.
\end{abstract}

Conclusion: Therefore, this study describes an efficient method to produce adequate amounts of biologically active canine IL-12, useful for immunomodulation studies in dogs.

Keywords: Interleukin-12, Dog, Protein expression optimization, Baculovirus

\section{Background}

Interleukin-12 was originally described as natural killer cell stimulatory factor-NKSF for its ability to promote activation of NK cells [1]. This cytokine plays an important role in inducing IFN- $\gamma$ production by $\mathrm{T}$ and natural killer cells [1,2], and in differentiation of Th1 CD4 $\pm \mathrm{T}$ cells $[3,4]$. Studies have shown that IL-12 has a potential therapeutic benefit in cancer $[5,6]$, infectious and inflammatory diseases, and as a vaccine adjuvant [7-9]. Thus, production of recombinant IL-12 has raised great interest in the scientific community.

\footnotetext{
*Correspondence: cpinheiro@posdoc.bahia.fiocruz.br;

ggileno@bahia.fiocruz.br

${ }_{1}^{1}$ Centro de Pesquisas Gonçalo Moniz, Fundação Oswaldo Cruz, Salvador,

Bahia, Brazil

Full list of author information is available at the end of the article
}

Interleukin-12 (IL-12) is a $70 \mathrm{kDa}$ heterodimeric cytokine composed of two disulfide-bonded subunits$\mathrm{p} 40$ and p35, [10-12]. The heterodimer (p70) is required for the biologic activity of IL-12 [13, 14]. In addition, only the posttranslational $\mathrm{N}$ glycosylated protein isoforms are biologically active [15]. Therefore, recombinant IL-12 should be produced in eukaryotic cells to display functional activity.

Okano et al. [16] cloned both subunits of canine IL-12 and described their expression in mammalian cells. Later, canine IL-12, showing biological activity, was cloned and expressed as a single chain protein (rsccalL-12) in the green monkey cell line COS-7 cells. rsccalL-12 was able to induce IFN- $\gamma$ mRNA expression in peripheral blood mononuclear cells (PBMC) from healthy and visceral leishmaniasis sick dogs [17]. However, the production of tens of milligrams of recombinant proteins in 
mammalian cells, necessary for pre-clinical trials in dogs, would be costly and laborious.

A feasible alternative for producing adequate amounts of the recombinant protein for pre-clinical trials is the baculovirus-insect cell system, which is being used extensively to express heterologous genes in insect cells cultures or insect larvae [18]. This system allows for production of high levels of recombinant proteins with posttranslational modifications, including N-glycosylation. Recombinant IL-12 subunits, p35 and p40, of bovine [19] and equine [20] have been produced in insect cells, resulting in a bioactive IL-12 p70 heterodimer. Furthermore, Poot et al. [21] have produced canine IL-12 in baculovirus-insect cell system using classical recombinant baculovirus and adherent insect cell cultures. However, this report lacks detailed information regarding production and yield of canine IL- 12 .

This study was conducted to determine appropriate conditions to produce moderate amounts of canine IL-12 to assess in a near future the feasibility of inducing Th1 immune responses in dogs, which would be useful in developing vaccines and treating diseases caused by intracellular pathogens, allergic diseases, and cancers. Herein, to determine appropriate conditions to produce IL-12 canine in tens of milligrams, several factors were evaluated to optimize the production of recombinant canine IL-12 in the baculovirus-insect cell system, as follows, native IL-12 versus the codon optimized IL-12 construct (both as a single-chain protein), the classical and a modified baculovirus vector, multiplicity of infection and time of infection. In addition, it is described a simple and an effective method to semi-quantitatively evaluate expression of secreted recombinant proteins in the baculovírus insect cell system (a dot-blot assay).

\section{Methods}

\section{Insect cell lines and culture}

Spodoptera frugiperda and Trichoplusia ni insect cell lines, Sf-9 and BTI-TN-5B1-4 (also called High-five), respectively, (Invitrogen, Carlsbad, USA) were used. Sf-9 cells were maintained in TNM-FH medium (Sigma Aldrich, St. Louis, USA) containing $10 \%$ fetal bovine serum (FBS, Invitrogen), $0.1 \%$ Pluronic F-68 solution (Sigma Aldrich), and $10 \mu \mathrm{g} / \mathrm{mL}$ gentamycin sulfate (Sigma Aldrich) (complete TMN-FH medium). High-five cells were cultured in Express-five serum-free medium (SFM) (Invitrogen) supplemented with $16 \mathrm{mM}$ of L-glutamine (Invitrogen), and $10 \mu \mathrm{g} / \mathrm{mL}$ gentamycin (complete Express-five SFM medium). Cell cultures were carried out at $27{ }^{\circ} \mathrm{C}$ either as a monolayer or in suspension in shaker flasks, according to the manufacturer's instructions.

\section{Interleukin-12 constructs}

Two DNA constructs encoding canine IL-12 single-chain protein (sccaIL-12 and sccalL-12opt, and Fig. 1) were cloned into the pFastBac1 plasmid (Invitrogen). The sccalL-12 construct was originally cloned from native canine mRNA into the pcDNA3.1 plasmid [17] while sccalL-12opt was synthesized with optimized codons for expression in T. $n i$ by Geneart GmBH (Regensburg, Germany). The recombinant proteins encoded by the constructs sccalL-12 and sccalL-12opt were named rsccaIL-12S and rsccalL-12L, corresponding to the length (short or long) of the polypeptide spacer between the $\mathrm{p} 40$ and p 35 chains, respectively. The successful introduction of inserts into the plasmid pFastBac1, generating the following constructs pFastBac1-sccalL-12 and pFastBac1sccalL12opt, was determined by DNA sequencing.

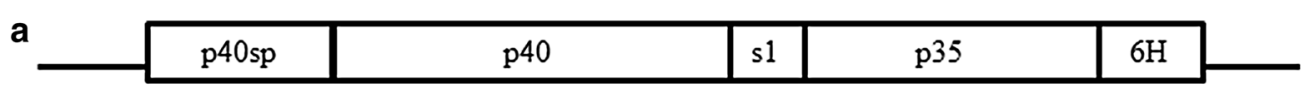

b

\begin{tabular}{|l|l|l|l|l|l|}
\hline $\mathrm{gp} 67 \mathrm{sp}$ & $\mathrm{p} 40$ & $\mathrm{~s} 2$ & $\mathrm{p} 35$ & $\mathrm{~s} 3$ & $6 \mathrm{H}$ \\
\hline
\end{tabular}

Fig. 1 Schematic diagram of DNA constructs encoding canine IL-12 single-chain protein. The construct with canine native DNA is composed in tandem by nucleotide sequences encoding: signal peptide of p40, p40 mature protein, spacer, p35 mature protein and His-tag (a); the construct with DNA optimized for Trichoplusia ni translation is composed in tandem by nucleotide sequences encoding: GP64 signal peptide of AcMNPV, p40 mature protein, a spacer, p35 mature protein, spacer and His-tag (b) 


\section{Generation of baculovirus encoding canine IL-12}

The inserts sccalL-12 and sccalL-12opt were transposed from the pFastBac1 plasmid constructs to: (a) Autographa californica multiple nuclear polyhedrosis virus (AcMNPV) bacmid [22] (bacmid named AcBac, Bac-to-Bac system, Invitrogen, cat. 10360-014) and (b) a chitinase and v-cathepsin knockout bacmid (bacmid called AcBac $\triangle \mathrm{CC}$, previously described by Kaba and collaborators) [23], using DH10Bac and DH10Bac $\Delta C C$ Escherichia coli strains, respectively. Recombinant AcBac-sccall-12, AcBac-sccall-12opt, AcBac $\Delta C C$ sccalL-12 and AcBac $\Delta$ CC-sccalL-12opt baculovirus constructs were obtained by transfecting Sf- 9 cells with the corresponding bacmids using a Lipofectamine reagent, following the manufacturer's instructions (Invitrogen). Baculovirus constructs with the insert from empty pFastBac1 plasmid (Invitrogen) were used as negative controls (AcBac-pFast-cont and AcBac $\Delta C C$-pFast-cont). These controls were obtainedthe cell-free $\mathrm{SN}$ were stored by transposing the DNA segment between Tn7R and Tn7L from the pFastBac1 plasmid into either the AcBac or AcBac $\Delta C C$ bacmid and then transfecting Sf-9 cells. Six days after transfection, Sf- 9 cell culture suspensions were spun down at $500 \times g$ for $5 \mathrm{~min}$ and baculovíruscontaining $\mathrm{SN}$ were stored protected from light at $4{ }^{\circ} \mathrm{C}$. To obtain high-titer viral stocks, log phase growing Sf-9 cells $\left(2 \times 10^{6} / \mathrm{mL}\right)$ cultured in suspension were infected at a multiplicity of infection (MOI) of 0.1 , either for $72 \mathrm{~h}$ or until cell viability decreased to approximately $75 \%$, and then the cell supernatants (SN) were collected as described above. Baculovirus stocks were titrated using the end-point dilution assay [24].

\section{Optimization of recombinant canine IL-12 expression by insect cells}

High-five cells $\left(1 \times 10^{6} /\right.$ well $)$ cultured as monolayers in serum free medium in 6-well titration plates were infected with the recombinant baculovirus constructs (AcBac-sccalL-12, AcBac-sccalL-12opt, AcBac $\Delta$ CCsccalL-12, or AcBac $\Delta$ CC-sccalL-12opt) at MOI of 2, 5 or 10 . To assess secreted recombinant protein, the cell culture SN were collected at 24,48 and $72 \mathrm{~h}$ post infection, floating cells were spun down at $500 \times g$ for $5 \mathrm{~min}$, and the cell-free $\mathrm{SN}$ were stored at $-20{ }^{\circ} \mathrm{C}$ until use. Recombinant canine IL-12 in the cell culture SN was evaluated by dot-blot assay, using mouse anti-histidine $\mathrm{C}$-terminal antibodies (mouse anti-His-C Ab, Invitrogen) as described below.

\section{Dot-blot and Western blot analysis}

For dot-blot assays, nitrocellulose membranes placed in a blotter device (BioRad, Hercules, United States) were directly coated with proteins from cell culture SN at 150 $\mu \mathrm{L} /$ well. The membranes were blocked with tris-buffered saline, $\mathrm{pH} 7.6$, containing $5 \%$ powdered non-fat milk and $0.05 \%$ Tween 20 and developed with mouse anti-HisC Ab (Invitrogen) diluted 1:5.000, goat anti-mouse IgG alkaline phosphatase-conjugate diluted 1:500 (SigmaAldrich), and alkaline phosphatase substrate (5-bromo4-chloro-3-indolyl-phosphate and nitro blue tetrazolium, Sigma Aldrich). For Western blot analysis, samples of SN or cell lysate from similar volumes of cultures of Highfive cells infected with AcBac-pFast-cont, AcBac $\Delta C C$ pFast-cont, AcBac-sccall-12, AcBac $\Delta$ CC-sccall-12, AcBac-sccalL-12opt or AcBac $\Delta C C$-sccalL-12opt baculovirus contructs at a MOI 5 for $48 \mathrm{~h}$ were fractioned by polyacrylamide gel electrophoresis with dodecyl sodium sulfate (SDS-PAGE) and then transferred to nitrocellulose membranes (BioRad). Detection of his-tagged protein was performed as described in the dot-blot assay.

Production of recombinant canine IL-12 by High-five cells Production of recombinant canine IL-12 in High-five cells was carried out in $50 \mathrm{~mL}$ suspension cultures using complete Express-five-SFM. For this, exponentially growing cells were adjusted to $2 \times 10^{6} / \mathrm{mL}$ and then infected with AcBac $\Delta$ CC-calL-12opt at MOI 5. After $48 \mathrm{~h}$ of infection, cell suspension was centrifuged at $3.000 \times g$ for $15 \mathrm{~min}$, cell-free SN was spun down at $30.000 \times g$ for $1 \mathrm{~h}$ to remove the virus particles and, the resulting $\mathrm{SN}$ was stored at $-70{ }^{\circ} \mathrm{C}$ until use. The virus-free $\mathrm{SN}$ was dialyzed twice against $0.15 \mathrm{M}$ phosphate-buffered saline, pH 7.8 (PBS) and once against binding buffer $(20 \mathrm{mM}$ $\mathrm{Na}_{2} \mathrm{HPO}_{4}, 500 \mathrm{mM} \mathrm{NaCl}, 20 \mathrm{mM}$ imidazole, $\mathrm{pH}$ 7.8), and then applied onto Histrap HP columns (GE Healthcare, Uppsala, Sweden) attached to an AKTA Purifier chromatography system (GE Healthcare). The column was washed with binding buffer and eluted using an imidazole linear gradient from 20 to $500 \mathrm{mM}$ in $20 \mathrm{mM}$ $\mathrm{Na}_{2} \mathrm{HPO}_{4}, 500 \mathrm{mM} \mathrm{NaCl}, \mathrm{pH}$ 7.8, buffer, following the manufacturer's recommendations. The purified protein (rsccalL-12L) was buffer exchanged to PBS using a Hitrap desalting column (GE Healthcare) and stored at $-70{ }^{\circ} \mathrm{C}$ until use. Protein samples were assessed by SDS-PAGE using $12 \%$ polyacrylamide gels and previously described Western blotting assay. A molecular weight calibration curve was prepared and molecular weight sizes of protein bands were estimated using GraphPad Prism v.5.0 software (GraphPad Prism Inc., San Diego, CA). In addition, the protein and endotoxin concentrations were determined using Micro BCA protein assay kit (Thermo Scientific, Rockford, USA, cat. 23235) and Limulus amebocyte lysate method (Lonza, Walkersville, USA, cat. 50-647U), respectively. 


\section{Circular dichroism measurements}

Circular dichroism spectra of rsccalL-12L were obtained at $0.7 \mathrm{mg} / \mathrm{mL}$ protein concentration. Far-UV spectra were measured from 190 to $260 \mathrm{~nm}$, averaged over three scans at a speed of $50 \mathrm{~nm} / \mathrm{min}$, and collected in $1 \mathrm{~nm}$ steps. The buffer baselines were subtracted from their respective sample spectra. These measurements were carried using a Jasco J-815 spectropolarimeter (Jasco Corp., Tokyo, Japan) with a $0.2 \mathrm{~cm}$ path-length quartz cuvette.

\section{Fluorescence spectroscopy measurements}

Tryptophan fluorescence emission spectra were obtained and recorded by setting the excitation wavelength at $280 \mathrm{~nm}$ and the emission spectrum was recorded from 295 to $415 \mathrm{~nm}$ using a Jasco FP-6500 spectrofluorimeter (Jasco Corp.).

\section{Assessment of interferon gamma (IFN- - ) production by peripheral blood mononuclear cells (PBMC) stimulated with purified recombinant canine IL-12}

Peripheral blood samples collected from six healthy adult mongrel dogs (three males and three females) were used. These animals were donated while still puppies to Gonçalo Moniz Research Center kennel by their owners. Thereafter the animals were housed at Gonçalo Moniz Research Center kennel. The experiments were performed in accordance with the guidelines of the Oswaldo Cruz Foundation for laboratory animal use and approved by Gonçalo Moniz Research Center Ethical Committee (Licence Protocol 021/2011-CPqGM). The concentration of IFN- $\gamma$ was measured in peripheral blood mononuclear cells (PBMC) culture SN by capture ELISA using a protocol previously described by Pereira and collaborators [27]. Briefly, PBMC purified in a Ficoll-Paque PLUS (GE Healthcare) gradient were suspended in RPMI-1640 medium (Sigma Aldrich) supplemented with $10 \%$ FBS, $10 \mathrm{mM}$ HEPES (Invitrogen), $\mathrm{pH}$ 7.0, $2 \mathrm{mM}$ L-glutamine (Sigma Aldrich) and $50 \mu \mathrm{g} / \mathrm{mL}$ gentamycin (complete RPMI medium). One hundred microliters of PBMC suspension at $2 \times 10^{5} / \mathrm{mL}$ were placed per well in a 96-well flat-bottom microtritation plate. One hundred $\mu \mathrm{L} /$ well of the following solutions were added to four wells: (a) complete RPMI, (b) SN of culture of COS-7 cells transfected with a plasmid encoding canine IL-2 (pcDNA3.1-caIL-2) [27] diluted 1:10 (COS-7 cells caIL-2 SN), (c) rsccaIL-12L at $40 \mathrm{ng} / \mathrm{mL}$, or (d) rsccalL- $12 \mathrm{~L}$ at $40 \mathrm{ng} / \mathrm{mL}$ in combination with COS-7 cells caIL-2 SN diluted 1:10. Then PBMC were cultured in a humidified atmosphere with $5 \% \mathrm{CO}_{2}$, at $37{ }^{\circ} \mathrm{C}$, for $48 \mathrm{~h}$. After that, supernatants of each quadruplicate were pooled, spun down at $300 \times g$ for 5 min to remove the cells and stored at $-20{ }^{\circ} \mathrm{C}$ until use. The concentration of IFN- $\gamma$ in the $\mathrm{SN}$ was determined by capture ELISA using specific antibodies produced by R\&D Systems (Minneapolis, USA), according to the manufacturer's instructions. A calibration curve was prepared with recombinant IFN- $\gamma$ (R\&D Systems) following the manufacturer's instructions and GraphPad Prism v.5.0 software.

\section{Statistical analysis}

The values for IFN- $\gamma$ concentration were compared by Repeated Measures ANOVA followed by Dunnett's posttest. Values of $p<0.05$ were considered significant. The statistical analysis was performed and graphs made using GraphPad Prism v.5.0 software.

\section{Results}

\section{Expression of recombinant canine IL-12}

To confirm transfer of the inserts (sccalL-12, sccalL12opt, and the segment between Tn7R and Tn7L from empty vector) from pFastBac1 plasmid constructs or pFastBac1 empty plasmid to either AcBac or AcBac $\Delta C C$ bacmid, PCR was carried with primers flanking the bacmid transposition sites. Amplicons produced by PCR showed the expected size (data not shown), indicating success in obtaining the recombinant (AcBac-sccalL-12, AcBac $\Delta$ CC-sccalL-12, AcBac-sccalL-12opt, AcBac $\Delta$ CCsccalL-12opt) and negative control (AcBac-pFast-cont, and AcBac $\Delta C C$-pFast-cont) bacmids.

To determine the best conditions for recombinant canine IL-12 expression in insect cells, High-five cells were infected with each of the four recombinant baculovirus constructs at MOI 2, 5 and 10, or one of the negative controls at MOI 10, and culture SN were assessed by dot-blot analysis at 24,48 and $72 \mathrm{~h}$ post-infection (pi), using an antibody specific for $\mathrm{C}$-terminal his-tags. Taking into account the background signal observed when $\mathrm{SN}$ of High-five cells cultured with AcBac-pFast-cont baculovirus was utilized at MOI 10 (Fig. 2), cells infected with recombinant baculovirus constructs started producing recombinant canine IL-12 after $48 \mathrm{~h}$ pi. Interestingly, the largest amounts of cytokine were secreted when the Highfive cells were infected with the AcBac-sccalL-12opt and AcBac $\Delta C C$-sccalL-12opt baculovirus constructs, whose inserts had codons optimized for expression in Trichoplusia $n i$, at MOI $272 \mathrm{~h} \mathrm{pi}$, MOI 548 or $72 \mathrm{~h} \mathrm{pi}$, and MOI 10 48 or $72 \mathrm{~h}$ pi (Fig. 2). High-five cells also synthesized the recombinant protein when infected with AcBac-sccalL-12 and AcBac $\triangle C C$-sccalL-12 constructs, but at a lower level comparing to the cells exposed to baculovirus constructs carrying the codon-optimized insert (AcBac-sccalL-12opt and AcBac $\Delta C C$-sccall-12opt) (Fig. 2). High-five cells infected with AcBac-sccalL-12, AcBac $\Delta$ CC-sccalL-12, AcBac-sccalL-12opt, and AcBac $\Delta C C$-sccalL-12opt baculovirus constructs at MOI 5 for $48 \mathrm{~h}$ were selected as the conditions for further studies. 


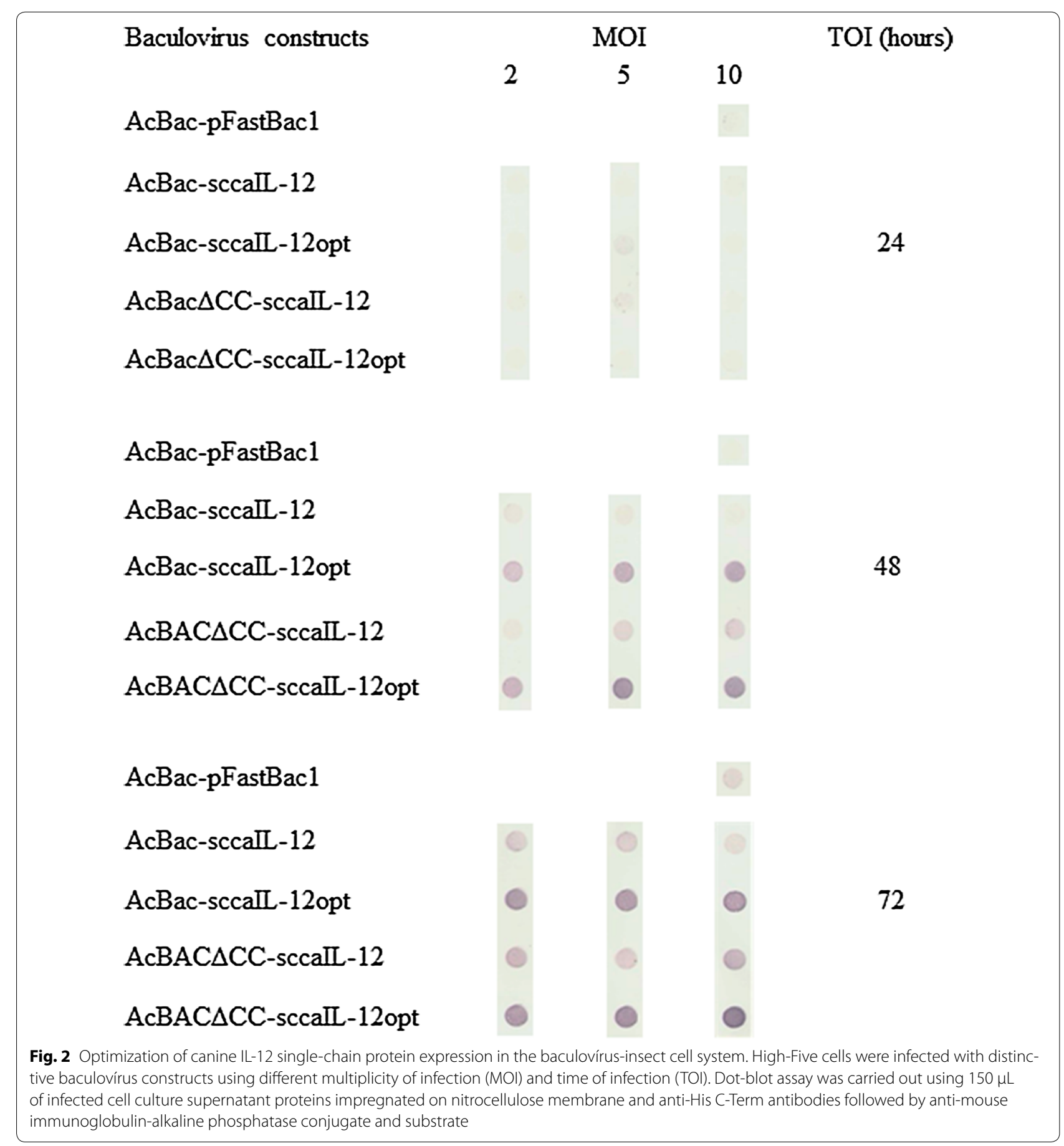

Baculovirus constructs containing sccalL-12opt promote efficient production of recombinant canine IL-12 by High-five cells

To assess the efficiency of secretion of recombinant canine IL-12, cell culture SN and lysates of High-five cells infected with either AcBac-sccalL-12, AcBac $\Delta C$ Csccall-12, AcBac-sccall-12opt, AcBac $\Delta C C$-sccall12opt, AcBac-pFast-cont or AcBac $\Delta C C$-pFast-cont baculovirus constructs at MOI 5 for $48 \mathrm{~h}$ were evaluated by Western blot with anti-His C-term antibodies.

In Western blot analysis, culture $\mathrm{SN}$ of cells infected with AcBac-sccalL-12 or AcBac $\Delta$ CC-sccalL- 12 baculovirus construct showed a protein band with a molecular mass of $67 \mathrm{kDa}$ (Fig. 3a, b, lanes 2), which was absent in the negative control SN (Fig. 3a, b, lanes 1). In addition, the culture SN of cells infected with either the 


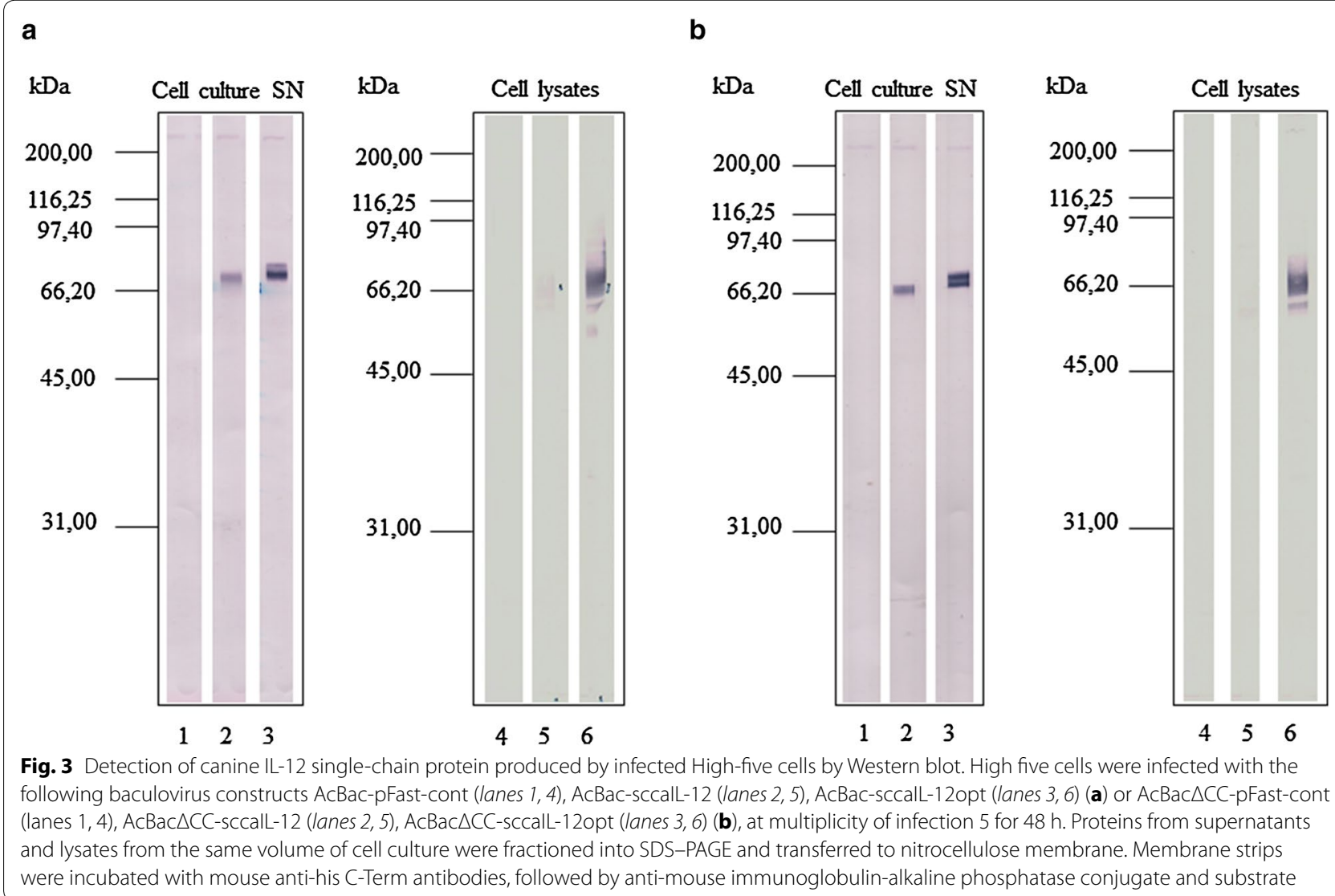

AcBac-sccalL-12opt or the AcBac $\Delta C C$-sccalL-12opt baculovirus construct showed two strong bands with molecular masses of 67 and $70 \mathrm{kDa}$, being these bands strongest when AcBac $\Delta \mathrm{CC}$-sccalL-12opt construct was used (Fig. 3a, b, lanes 3). Therefore, the amount of IL-12 detected in the $\mathrm{SN}$ of cells infected with either baculovirus construct carrying the codon optimized insert (AcBac-sccalL-12opt and AcBac $\Delta$ CC-sccalL-12opt) was higher than in the $\mathrm{SN}$ of cells cultured with baculovirus constructs carrying native canine IL-12 cDNA (AcBacsccalL-12 and AcBac $\Delta$ CC-sccall-12).

Analyzed by Western blot, lysates of cells infected with AcBac-sccalL-12 or AcBac $\Delta$ CC-sccalL-12 showed a weak broad band of average molecular mass of $67 \mathrm{kDa}$ and a weak band of $61 \mathrm{kDa}$, respectively, which were not seen -in negative control lysates. Lysates of cells infected with AcBac-sccalL-12opt and AcBac $\Delta C C$-sccalL-12opt baculovirus constructs displayed a strong broad band with an average molecular mass of $67 \mathrm{kDa}$ and a weak band of $61 \mathrm{kDa}$ (Fig. 3), which were not present in the negative control lysates. In addition, lysate of High-five cells infected with AcBac-sccalL-12opt presented a weak band of $54 \mathrm{kDa}$ (Fig. 3), absent in the corresponding negative control lysate.
Western blot results suggest that the AcBac $\Delta C C$ sccalL-12opt recombinant baculovirus construct induced the highest amount of secreted recombinant canine IL-12 (rsccalL-12L) and more than half of the total recombinant protein produced was retained within the cells.

To determine the yield and biological activity, rsccaIL-12L was purified from the SN of High-Five cells infected with AcBac $\Delta C C$-sccalL-12opt baculovirus construct at MOI 5 for $48 \mathrm{~h}$. After purification from the cell SN by affinity chromatography, rsccalL-12L displayed a single band with molecular weight size of $68.5 \mathrm{kDa}$ in Western blot and SDS-PAGE analysis (Fig. 4). Considering the amount of the recombinant protein purified from $50 \mathrm{~mL}$ of cell culture $\mathrm{SN}$, it was estimated a yield of $6 \mathrm{mg}$ of rsccalL-12L per liter of culture. Purified rsccalL-12L had $0.1 \mathrm{EU}$ of endotoxin per mg of protein.

The fluorescence spectrum of rsccalL-12L showed maximum peak in $338 \mathrm{~nm}$ (Fig. 5a), suggesting that the recombinant protein was properly folded. In addition, circular dichroism analysis revealed a spectrum with a maximum negative ellipticity at $210 \mathrm{~nm}$, indicating that the target protein has a predominantly $\beta$-sheet structure (Fig. 5b). 


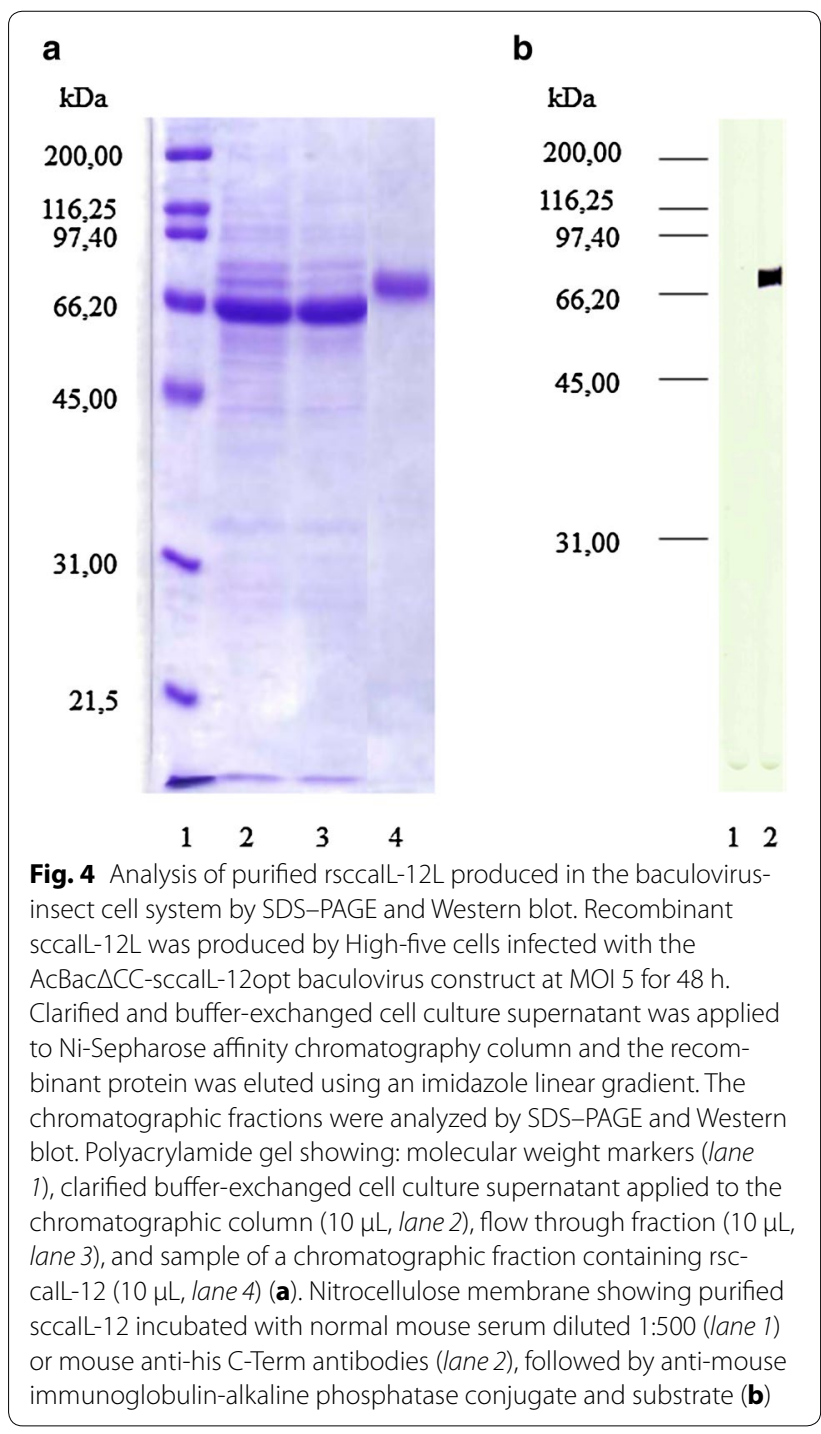

Recombinant canine sccalL-12L produced by High-five cells induces IFN- $\gamma$ in homologous PBMC

The biological activity of rsccalL- 12 was evaluated by testing its ability to induce IFN- $\gamma$ production in PBMC from six healthy adult dogs. Canine PBMC were cultured for $48 \mathrm{~h}$ with:(a) complete RPMI, (b) COS-7 cells caIL-2 SN, (c) rsccalL-12L, or (d) rsccalL-12L in combination with COS-7 cells calL-2 SN. IFN- $\gamma$ was measured in culture SN by capture ELISA. Canine PBMC have not produced IFN- $\gamma$ when cultured in complete $\mathrm{RPMI}$ (mean $\pm \mathrm{SD}, 1 \pm 1 \mathrm{pg} / \mathrm{mL}$ ) or in the presence of SN COS-7 containing IL-2 $(1 \pm 2 \mathrm{pg} / \mathrm{mL})$. PBMC of 3 out of 6 dogs produced IFN- $\gamma$ when cultured with rsccaIL-12L at $20 \mathrm{ng} / \mathrm{mL}$, however, the mean concentration (mean $\pm \mathrm{SD}, 38 \pm 40 \mathrm{pg} / \mathrm{mL}$ ) observed in the SN of stimulated cells was not significantly different from the SN of cells cultured in medium alone (Fig. 6). Nevertheless, when PBMC were stimulated with both rsccalL-12L at $20 \mathrm{ng} / \mathrm{ml}$ and SN COS-7 containing IL-2, cells of 6 out of 6 dogs produced significantly higher amounts of IFN- $\gamma$ $(247 \pm 134 \mathrm{pg} / \mathrm{mL})$ than cells cultured with complete RPMI (Fig. 6), indicating that purified rsccalL-12L was biologically active.

\section{Discussion}

IL-12 has the potential to promote, alone or in combination with other immunomodulatory agents, cell-mediated immune responses [9, 25-27], which are useful in the control of infections caused by intracellular pathogens, of allergic diseases mediated by antibody [28], and of neoplasms [26]. Aiming at optimizing conditions to produce tens of milligrams of canine IL-12 for future preclinical trials in dogs, several parameters were evaluated in the present work, including different baculovirus constructs, and time of infection (TOI 24, 48 and $72 \mathrm{~h}$ ) and multiplicity of infection (MOI 2, 5 and 10) of High-five insect cells.

In order to obtain a good yield of secreted mammalian recombinant protein in the baculovirus-insect cell system, DNA constructs have been used with: (a) elements potentially capable of promoting translation or secretion or (b) baculovirus vector framework knocked out of genes encoding proteins targeted to endoplasmic reticulum. For example, to enhance translation, constructs have been made with: (a) AT rich nucleotide sequence before the initiation codon and an adenine at position +4 , that would promote recognition of mRNA by ribosomes in insect cells, instead of Kozak sequence [29-31], (b) codons specifically optimized for the species of the used host [32]. To improve secretion, constructs have been prepared with: (a) signal peptide encoding sequences from proteins efficiently targeted to endoplasmic reticulum of insect cells, like AcMNPV GP64 protein or honeybee venom melittin, instead of native signal peptide [31, 33], or (b) a baculovirus vector from which several genes were deleted, for example, chitinase, cathepsin, p10, p26 and p74 [23, 34-36], which encode proteolytic enzymes or proteins capable of competing for secretory machinery resources of the host cell.

In the current study, a recombinant baculovirus construct (AcBac-sccalL-12) was used with the classic baculovirus vector framework (AcBac) and canine native DNA encoding IL-12 (sccalL-12). In addition, recombinant baculovirus constructs were used with: (a) AcBac knockout of chitinase and cathepsin genes (AcBac $\triangle C C$ ) and sccaIL-12 insert (AcBac $\Delta$ CC-sccalL-12), (b) AcBac and an insert comprised of DNA encoding AcMNPV GP64 signal peptide and canine IL-12 mature polypeptides, with optimized codons for translation in T. ni (sccalL-12opt) (AcBac-sccalL-12opt) and, (c) AcBac $\Delta C C$ 

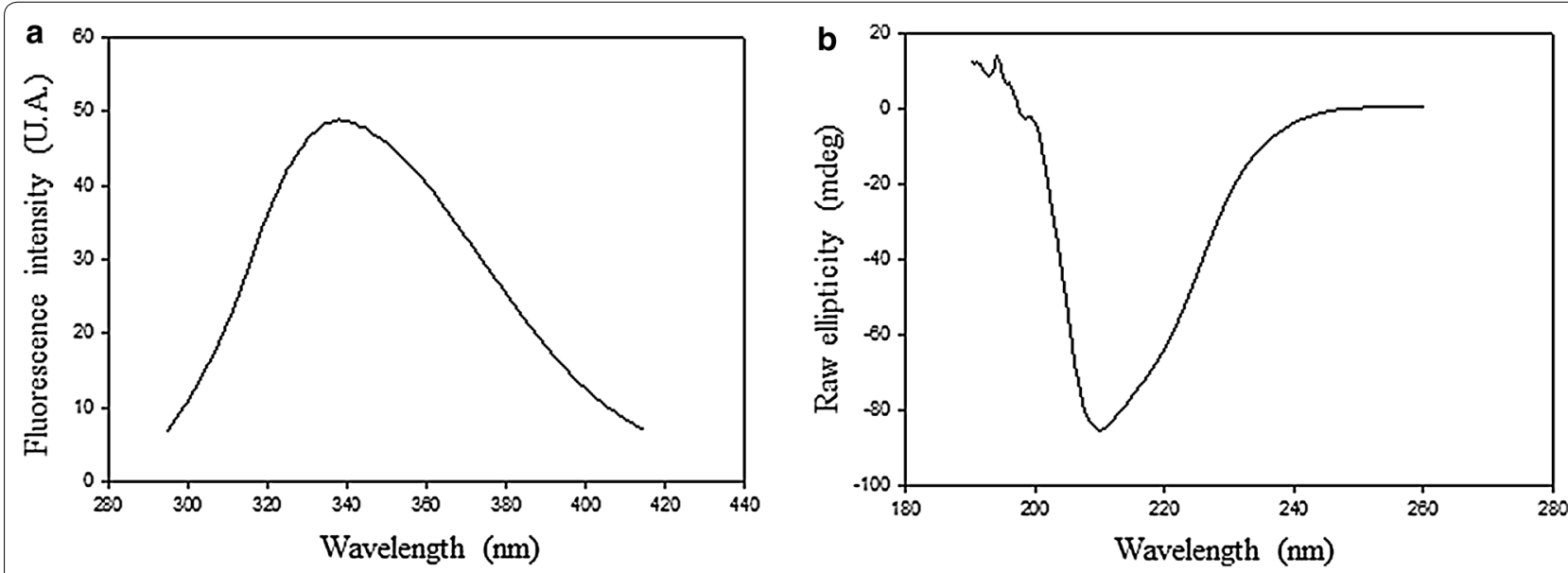

Fig. 5 Fluorescence and circular dichroism of rsccalL-12L. Intrinsic fluorescence spectra of rsccalL-12L obtained by excitation at $280 \mathrm{~nm}$, and emission from 295 to $415 \mathrm{~nm}$ (a). Circular dichroism spectra of rsccalL-12L, at 190-260 nm, resulting from three consecutive measurements at the speed of $50 \mathrm{~nm} / \mathrm{min}$ (b)

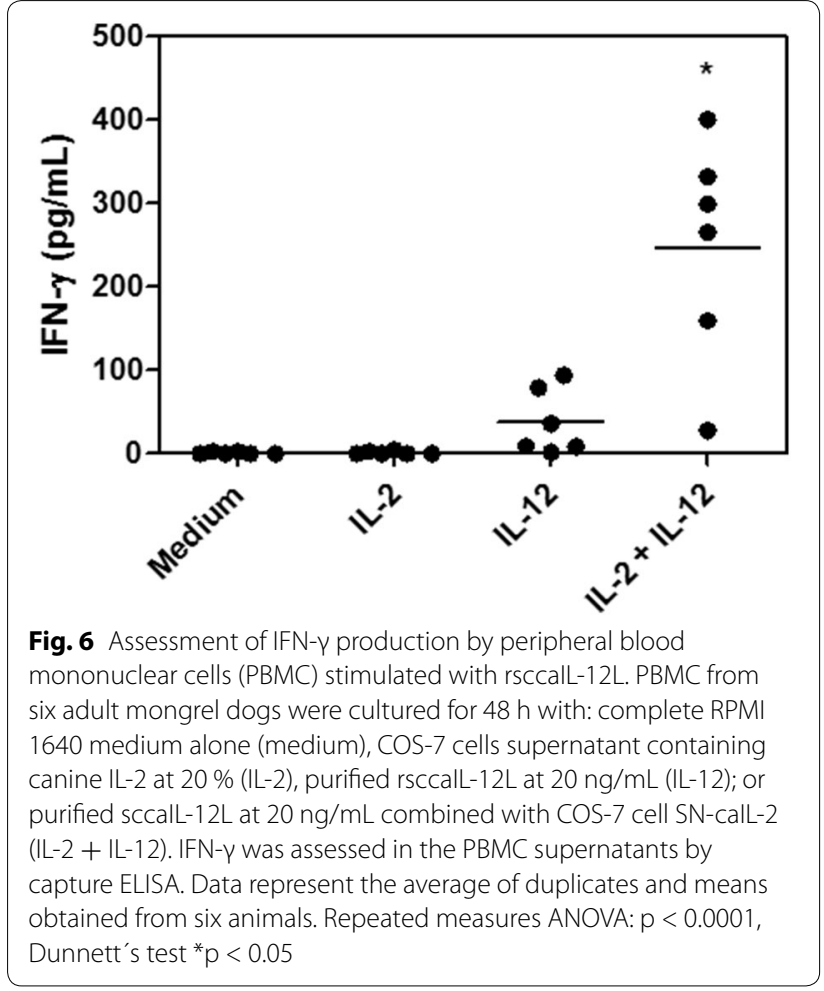

and sccalL-12opt (AcBac $\Delta$ CC-sccalL-12opt). Furthermore, constructs were assessed by their ability to promote IL-12 secretion in High-Five cells to determine if any of the changes made in basic construct design was advantageous.

The constructs were evaluated initially by assessing culture SN of High Five cells infected with MOI 2, 5 or 10 and
TOI 24,48 or $72 \mathrm{~h}$, using the dot-blot assay. All baculovirus constructs were able to induce the secretion of both rsccaIL-12S and rsccalL-12L recombinant proteins. However, AcBac-sccalL-12opt and AcBac $\Delta$ CC-sccalL-12opt constructs induced the largest quantities of rsccalL-12Lthe latter being slightly more efficient than the former. These results suggest that the use of: (a) AcMNPV GP64 signal peptide, in contrast to the native signal peptide, and/or (b) codons optimized for T. ni (in contrast to the canine native codons), and (c) a baculovirus vector devoid of cathepsin and chitinase genes (in contrast to the classical baculovirus vector), contributed to increased production of secreted recombinant protein. Factors described in "a" and " $\mathrm{b}$ " had a greater impact than the one mentioned in "c". Interestingly, in one experiment in which production of secreted recombinant canine IL-12 was assessed by dot-blot of the supernatant of Sf-9 cells infected with MOI 2, 5 and 10 and TOI of 24, 48 and $72 \mathrm{~h}$, the four baculovirus constructs were equally efficient (data not shown), suggesting that replacement of the native signal peptide for that of AcMNPV GP64, optimizing codons for T. $n i$ and the use of a baculovirus vector devoid of cathepsin and chitinase genes have no impact on expression in Sf-9 cells. These results are in agreement with reports by several authors which show that certain changes in the insert and in the genome of the baculovirus vector itself can promote an increased yield of recombinant proteins and that the same baculovirus construct may promote different yields in different host cells, e.g., Sf9 and High-Five cells [37-39]. Therefore, the baculovirus-insect cell system is not fully predictable [33].

Various methods have been used for detection of secreted recombinant proteins in the process of 
optimizing production conditions in baculovirus-insect cell system, including ELISA [37, 39, 40], enzyme assay $[41,42]$ and Western blot analysis [23, 43, 44] - the latter being most commonly used. Western blot analysis is a labor-intensive method that involves several steps, including separation in polyacrylamide gel and transfer to nitrocellulose membrane, before protein detection. In the current study, a simpler method (dot-bot) was successfully used to optimize production conditions of a recombinant protein secreted in the culture $\mathrm{SN}$ of cells grown in serum-free medium. The best conditions for production of the recombinant protein were MOI 5 and TOI $48 \mathrm{~h}$. These conditions were chosen taking into account the difference between the signal to noise ratio observed in the dot-blot assay. In our laboratory, dot-blot analysis was also successfully used to optimize production of three other secreted recombinant proteins in the baculovirus-insect cell system (unpublished data).

To determine the secretion efficiency and integrity of the recombinant canine IL-12 produced by High-five cells infected with each of the four recombinant baculovirus constructs at the best conditions, Western blot assays were carried out using samples of the culture SN and cell lysates. Taking into account the presence and intensity of bands with molecular masses between 67 and $70 \mathrm{kDa}$ detected by anti-His tagged antibody, the total production (sum of the recombinant protein in culture SN and cell lysate) and secretion of the recombinant canine IL-12, it can be concluded that the recombinant protein was obtained in progressively larger amounts by the constructs AcBac-sccalL-12, AcBac $\Delta$ CC-sccall-12, AcBac-sccalL-12opt and AcBac $\Delta C C$-sccalL-12opt. Data concerning detection of the recombinant protein in culture $\mathrm{SN}$ corroborate the findings of the dot-blot assay, confirming that the use of the GP64 signal peptide and the codons optimized for $T$. $n i$ had the greatest impact and that the baculovirus vector framework devoid of cathepsin and chitinase genes impacted only modestly on the production of the secreted IL- 12 .

For unknown reasons, when the constructs AcBacsccalL-12opt and AcBac $\Delta$ CC-sccalL-12opt were used, apparently, most of the translated protein was retained inside the host cells (Fig. 3). A few studies have also shown that sometimes, especially while using High-Five cells, baculovirus constructs have the potential to induce secretion of tens to hundreds of milligrams of recombinant proteins per liter of cell culture [39, 45]. Since the yield of canine IL-12 observed in this study was lower than $10 \mathrm{mg} / \mathrm{L}$ of culture, it is improbable that the secretory machinery was overwhelmed to explain the retention of most of the recombinant cytokine within the host cells. Many authors have also observed retention of secretable recombinant protein inside the cells when using the baculovirus-insect cell system [23, 39, 46, 47]. The presence of a small number of bands detected in the cell lysate by Western blot, being the majority of bands detected with molecular masses above the predicted mass for the mature protein (rsccalL-12S, $58.3 \mathrm{kDa}$ and rsccalL-12L, $61.3 \mathrm{kDa}$ ), suggests that there was little, if any, proteolysis of the recombinant protein during the production process. The two bands $(67$ and $70 \mathrm{kDa})$ observed in culture SN of cells infected with AcBacsccalL-12opt and AcBac $\Delta$ CC-sccalL-12opt baculovirus constructs probably represent glycosylated isoforms of rsccalL-12L [23]. Assessment of glycosylation of rsccIL$12 \mathrm{~L}$ is underway.

In view of the results obtained from dot blot and Western blot assays, the baculovirus construct AcBac $\Delta C C$ sccalL-12opt was used to infect High-Five cells at MOI 5 and TOI 48 in order to produce recombinant canine IL-12 for purification and biological evaluation.

To determine the quality, samples of the purified protein were analyzed by SDS-PAGE, Western blot, fluorescence emission spectrum, circular dichroism, and for endotoxin contamination. Evaluation carried out by SDS-PAGE with gel stained with Coommassie blue and Western blotting showed a single band with an average molecular mass of $68.5 \mathrm{kDa}$, instead of the expected two bands of 67 and $70 \mathrm{kDa}$, previously detected during analysis of culture SN. This was probably related to the greater amount of protein used in the previous experiments making the bands appear fused. Assessment by the fluorescence emission spectrum and circular dichroism revealed data compatible with a folded protein predominantly composed of beta sheets. These findings are consistent with data previously reported for human IL-12 $[48,49]$. Endotoxin was detected only in very low concentrations. Finally, determination of protein concentration carried out in samples of two independent experiments allowed the estimation of a yield of 6-9 $\mathrm{mg}$ of sccalL12L per liter of insect cell culture. Therefore, taking into account protein purity, integrity, folding, endotoxin contamination, and yield, the recombinant protein was successfully purified.

The functional activity of the purified protein (rsccalL12L) was determined by its ability to induce the synthesis of IFN- $\gamma$ in canine PBMC. Previous studies reported the requirement of dual stimulation, using both IL-12 and IL-2, for the induction of IFN- $\gamma$ secretion by canine PBMC [27]. In the current study, PBMC from healthy dogs were cultured with rsccalL-12L alone or in combination with COS-7 SN containing IL-2, the latter at a concentration that was unable to induce IFN- $\gamma$ synthesis by itself. Stimulation with rsccalL-12L alone induced IFN- $\gamma$ production above background levels in 3 out of 6 dogs, although this 
was not statistically significant. On the other hand, stimulation with rsccalL-12L in combination with COS-7 SN containing IL-2 resulted in synthesis of IFN- $\gamma$ by PBMC from all six dogs tested, and compared with PBMC cultured with COS-7 SN containing IL-2 alone or medium alone, this was statistically significant, thus, indicating that the recombinant protein produced was biologically active. Previously, Poot et al. [21] have produced canine IL-12 in the baculovirus-insect cell system and shown it is able to induce proliferation of equine lymphoblasts. Unfortunately, these authors have not assessed the ability of canine IL-12 to stimulate the IFN- $\gamma$ synthesis in canine PBMC that would allow some comparison with the current work.

In conclusion, this is the first description of a method for production of bioactive canine IL-12 in suitable amounts for pre-clinical trials. In addition, the combination of several factors (use of AcMNPV GP64 signal sequence, codons optimized to insect cells, and baculovirus knockout for chitinase and cathepsin) described in the current manuscript has a wide application in the field of recombinant protein production, useful for future studies on prophylactic and therapeutic interventions in cancer, inflammatory and infectious diseases in dogs.

\begin{abstract}
Abbreviations
AcBac-pFast-cont: control classical baculovirus with no canine IL-12 insert; AcBac $\triangle C C$-pFast-cont: control chitinase A and v-cathepsin knockout baculovirus with no canine IL-12 insert; AcBac-sccall-12: classical baculovirus containing a native insert of canine IL-12; AcBac-sccalL-12opt: classical baculovirus containing optimized insert of canine IL-12; AcBac $\Delta C C$-sccall-12: chitinase $A$ and $v$-cathepsin knockout baculovirus containing a native insert of canine IL-12; AcBac $\triangle C C$-sccalL-12opt: chitinase A and v-cathepsin knockout baculovirus containing an optimized insert of canine IL-12; His: histidine; pFastBac1: empty vector; pFastBac1-sccall-12: vector containing a native insert of canine IL-12; pFastBac1-sccalL12opt: vector containing optimized insert of canine IL-12; sccall-12: native insert of canine IL-12 as single-chain protein; sccalL-12opt: optimized insert of canine IL-12 as single-chain protein; $\mathrm{SN}$ : supernatants.
\end{abstract}

\section{Authors' contributions \\ CGMP was involved in planning and carrying out all the experiments (except protein fluorescence and circular dichroism analysis of IL-12), data analysis and in manuscript writing. MOP was involved in carrying out some of the experiments (recombinant baculovirus generation and optimization assay). NCT was involved in carrying out some of the experiments (western blot assay). APDAB was involved in carrying out some of the experiments (protein fluorescence and circular dichroism analysis of IL-12). MMvO was involved in advice on baculovirus technology and manuscript writing. GGSO was involved in planning and carrying out some of the experiments. (optimization assay), data analysis and in manuscript writing. All authors read and approved the final manuscript.}

\footnotetext{
Author details

${ }^{1}$ Centro de Pesquisas Gonçalo Moniz, Fundação Oswaldo Cruz, Salvador, Bahia, Brazil. ${ }^{2}$ Programa Nacional de Pós Doutorado-CAPES/Programa de Pós-graduação em Biotecnologia em Saúde e Medicina Investigativa, Centro de Pesquisas Gonçalo Moniz, Fundação Oswaldo Cruz, Salvador, Bahia, Brazil. ${ }^{3}$ Laboratório de Macromoléculas, Bio-Manguinhos, Fundação Oswaldo Cruz, Rio de Janeiro, Brazil. ${ }^{4}$ Laboratory of Virology, Wageningen University, Wageningen, The Netherlands. ${ }^{5}$ Instituto Nacional de Ciência e Tecnologia de Doenças Tropicais (INCT-DT), Salvador, Brazil.
}

\section{Acknowledgements}

The authors would like to thank Dr. Peter Lydyard, University of Westminster, London, for providing English revision and the Program for Technological Development in Tools for Health-PDTIS-FIOCRUZ for the use of its facilities. This work was supported by the Conselho Nacional de Desenvolvimento Científico e Tecnológico (CNPq) and Instituto Nacional de Ciência e Tecnologia de Doenças Tropicais (INCT-DT).

\section{Competing interests}

The authors declare that they have no competing interests.

Received: 10 June 2015 Accepted: 8 January 2016

Published online: 22 January 2016

\section{References}

1. Kobayashi M, Fitz L, Ryan M, Hewick RM, Clark SC, Chan S, Loudon R, Sherman F, Perussia B, Trinchieri G. Identification and purification of natural killer cell stimulatory factor (NKSF), a cytokine with multiple biologic effects on human lymphocytes. J Exp Med. 1989;170(3):827-45.

2. Chan SH, Perussia B, Gupta JW, Kobayashi M, Pospisil M, Young HA, Wolf SF, Young D, Clark SC, Trinchieri G. Induction of interferon gamma production by natural killer cell stimulatory factor: characterization of the responder cells and synergy with other inducers. J Exp Med. 1991;173(4):869-79.

3. Manetti R, Parronchi P, Giudizi MG, Piccinni MP, Maggi E, Trinchieri G, Romagnani S. Natural killer cell stimulatory factor (interleukin 12 [IL-12]) induces T helper type 1 (Th1)-specific immune responses and inhibits the development of IL-4-producing Th cells. J Exp Med. 1993;177(4):1199-204.

4. Hsieh CS, Macatonia SE, Tripp CS, Wolf SF, O'Garra A, Murphy KM. Development of TH1 CD4 + T cells through IL-12 produced by Listeria-induced macrophages. Science. 1993;260(5107):547-9.

5. Brunda MJ, Luistro L, Warrier RR, Wright RB, Hubbard BR, Murphy M, Wolf SF, Gately MK. Antitumor and antimetastatic activity of interleukin 12 against murine tumors. J Exp Med. 1993;178(4):1223-30.

6. Chuang TF, Lee SC, Liao KW, Hsiao YW, Lo CH, Chiang BL, Lin XZ, Tao MH, Chu RM. Electroporation-mediated IL-12 gene therapy in a transplantable canine cancer model. Int J Cancer. 2009;125(3):698-707.

7. Afonso LC, Scharton TM, Vieira LQ, Wysocka M, Trinchieri G, Scott P. The adjuvant effect of interleukin-12 in a vaccine against Leishmania major. Science. 1994;263(5144):235-7.

8. Gurunathan S, Prussin C, Sacks DL, Seder RA. Vaccine requirements for sustained cellular immunity to an intracellular parasitic infection. Nat Med. 1998;4(12):1409-15.

9. Tewary P, Saxena S, Madhubala R. Co-administration of IL-12 DNA with rORFF antigen confers long-term protective immunity against experimental visceral leishmaniaisis. Vaccine. 2006;24(13):2409-16.

10. Gately MK, Desai BB, Wolitzky AG, Quinn PM, Dwyer CM, Podlaski FJ, Familletti PC, Sinigaglia F, Chizonnite R, Gubler U, et al. Regulation of human lymphocyte proliferation by a heterodimeric cytokine, IL-12 (cytotoxic lymphocyte maturation factor). J Immunol. 1991;147(3):874-82.

11. Wolf SF, Temple PA, Kobayashi M, Young D, Dicig M, Lowe L, Dzialo R, Fitz L, Ferenz C, Hewick RM, et al. Cloning of cDNA for natural killer cell stimulatory factor, a heterodimeric cytokine with multiple biologic effects on T and natural killer cells. J Immunol. 1991;146(9):3074-81.

12. Trinchieri G. Interleukin-12: a proinflammatory cytokine with immunoregulatory functions that bridge innate resistance and antigen-specific adaptive immunity. Annu Rev Immunol. 1995;13:251-76.

13. Gubler U, Chua AO, Schoenhaut DS, Dwyer CM, McComas W, Motyka R, Nabavi N, Wolitzky AG, Quinn PM, Familletti PC, et al. Coexpression of two distinct genes is required to generate secreted bioactive cytotoxic lymphocyte maturation factor. Proc Natl Acad Sci USA. 1991;88(10):4143-7.

14. Schoenhaut DS, Chua AO, Wolitzky AG, Quinn PM, Dwyer CM, McComas W, Familletti PC, Gately MK, Gubler U. Cloning and expression of murine IL-12. J Immunol. 1992;148(11):3433-40.

15. Murphy FJ, Hayes MP, Burd PR. Disparate intracellular processing of Human IL-12 preprotein. subunits: a typical processing of the P35 signal peptide1. J Immunol. 2000;164:839-47. 
16. Okano F, Satoh M, Yamada K. Cloning and expression of the cDNA for canine interleukin-12. J Interferon Cytokine Res. 1997;17:713-8.

17. Santos LR, Barrouin-Melo SM, Chang YF, Olsen J, McDonough SP, Quimby F, dos Santos WL, Pontes-de-Carvalho LC, Oliveira GG. Recombinant single-chain canine interleukin 12 induces interferon gamma mRNA expression in peripheral blood mononuclear cells of dogs with visceral leishmaniasis. Vet Immunol Immunopathol. 2004;98(1-2):43-8.

18. van Oers MM, Pijlman GP, Vlak JM. Thirty years of baculovirus-insect cell protein expression: from dark horse to mainstream technology. J Gen Virol. 2015;96(Pt 1):6-23.

19. Takehara K, Nagata T, Kikuma R, Takanashi T, Yoshiya S, Yamaga A, Yokomizo Y, Nakamura M. Expression of a bioactive bovine interleukin-12 using baculovirus. Vet Immunol Immunopathol. 2000;77(1-2):15-25.

20. McMonagle EL, Taylor S, van Zuilekom H, Sanders L, Scholtes N, Keanie LJ, Hopkins CA, Logan NA, Bain D, Argyle DJ, et al. Production of biologically active equine interleukin 12 through expression of p35, p40 and single chain IL-12 in mammalian and baculovirus expression systems. Equine Vet J. 2001;33(7):693-8.

21. Poot J, Spreeuwenberg K, Sanderson SJ, Schijns VECJ, Mottram JC, Coombs GH, Vermeulen AN. Vaccination with a preparation based on recombinant cysteine peptidases and canine IL-12 does not protect dogs from infection with Leishmania infantum. Vaccine. 2006;24:2460-8.

22. Luckow VA, Lee SC, Barry GF, Olins PO. Efficient generation of infectious recombinant baculoviruses by site-specific transposon-mediated insertion of foreign genes into a baculovirus genome propagated in Escherichia coli. J Virol. 1993;67(8):4566-79.

23. Kaba SA, Salcedo AM, Wafula PO, Vlak JM, van Oers MM. Development of a chitinase and $v$-cathepsin negative bacmid for improved integrity of secreted recombinant proteins. J Virol Methods. 2004;122(1):113-8.

24. O'Reilly DR, Miller LK, Luckow VA. Virus Methods. In: Baculovirus Expression Vectors: a laboratory manual. New York: Oxford University Press; 1994.

25. Saldarriaga OA, Perez LE, Travi BL, Melby PC. Selective enhancement of the type 1 cytokine response by expression of a canine interleukin (IL)-12 fused heterodimeric DNA. Vet Immunol Immunopathol. 2006;110(3-4):377-88.

26. Marchi LH, Paschoalin T, Travassos LR, Rodrigues EG. Gene therapy with interleukin-10 receptor and interleukin-12 induces a protective interferon-gamma-dependent response against B16F10-Nex2 melanoma. Cancer Gene Ther. 2011;18(2):110-22.

27. Pereira AM, Pinheiro CGM, Dos Santos LR, Teixeira NC, Chang YF, Pontesde-Carvalho LC, Oliveira GG. Requirement of dual stimulation by homologous recombinant IL-2 and recombinant IL-12 for the in vitro production of interferon gamma by canine peripheral blood mononuclear cells. BMC Res. Notes. 2014;7(1):460.

28. Gavett SH, O'Hearn DJ, Li X, Huang SK, Finkelman FD, Wills-Karp M Interleukin 12 inhibits antigen-induced airway hyperresponsiveness, inflammation, and Th2 cytokine expression in mice. J Exp Med. 1995;182(5):1527-36.

29. Kozak M. An analysis of 5'-noncoding sequences from 699 vertebrate messenger RNAs. Nucleic Acids Res. 1987;15(20):8125-48.

30. Chang MJ, Kuzio J, Blissard GW. Modulation of translational efficiency by contextual nucleotides flanking a baculovirus initiator AUG codon. Virology. 1999;259(2):369-83.

31. van Oers MM. Opportunities and challenges for the baculovirus expression system. J Invertebr Pathol. 2011;107(Suppl):S3-15.

32. Gao J, Meng C, Chen Z, Li C, Liu G. Codon optimization of the rabbit hemorrhagic disease virus (RHDV) capsid gene leads to increased gene expression in Spodoptera frugiperda 9 (Sf9) cells. J Vet Science. 2013;14(4):441-7.

33. Jarvis DL. Baculovirus-insect cell expression systems. Methods Enzymol. 2009:463:191-222.

34. Hitchman RB, Possee RD, Crombie AT, Chambers A, Ho K, Siaterli E, Lissina O, Sternard H, Novy R, Loomis K, et al. Genetic modification of a baculovirus vector for increased expression in insect cells. Cell Biol Toxicol. 2010;26(1):57-68.

35. Hitchman RB, Possee RD, Siaterli E, Richards KS, Clayton AJ, Bird LE, Owens RJ, Carpentier DC, King FL, Danquah JO, et al. Improved expression of secreted and membrane-targeted proteins in insect cells. Biotechnol Appl Biochem. 2010;56(3):85-93.

36. Metz SW, Geertsema C, Martina BE, Andrade P, Heldens JG, van Oers MM, Goldbach RW, Vlak JM, Pijlman GP. Functional processing and secretion of Chikungunya virus E1 and E2 glycoproteins in insect cells. Virol J. 2011;8:353.

37. Wickham TJ, Nemerow GR. Optimization of growth methods and recombinant protein production in BTI-Tn-5B1-4 insect cells using the baculovirus expression system. Biotechnol Prog. 1993;9(1):25-30.

38. Kim JS, Choi JY, Roh JY, Lee HY, Jang SS, Je YH. Production of recombinant polyhedra containing Cry1Ac fusion protein in insect cell lines. J Microbiol Biotech. 2007;17(5):739-44.

39. Furuta T, Ogawa T, Katsuda T, Fujii I, Yamaji H. Efficient production of an antibody fab fragment using the baculovirus-insect cell system. J Biosci Bioeng. 2010;110(5):577-81.

40. Golden A, Austen DA, van Schravendijk MR, Sullivan BJ, Kawasaki ES, Osburne MS. Effect of promoters and signal sequences on the production of secreted HIV-1 gp120 protein in the baculovirus system. Protein Express Purif. 1998;14(1):8-12.

41. Neutra R, Levi BZ, Shoham Y. Optimization of protein-production by the baculovirus expression vector system in shake flasks. Appl Microbiol Biotechnol. 1992;38(1):74-8.

42. Kato T, Murata T, Usui T, Park EY. Comparative analysis of GFP(UV)-beta1,3$\mathrm{N}$-acetylglucosaminyltransferase 2 production in two insect-cell-based expression systems. Protein Express Purif. 2004;35(1):54-61.

43. East J, De Sousa MA. The thymus autoimmunity and malignancy in New Zealand black mice. Natl Cancer Inst Monogr. 1966;22:605-16.

44. Wang YB, Wang ZY, Chen HY, Cui BA, Wang YB, Zhang HY, Wang R. Secretory expression of porcine interferon-gamma in baculovirus using HBM signal peptide and its inhibition activity on the replication of porcine reproductive and respiratory syndrome virus. Vet Immunol Immunopathol. 2009;132(2-4):314-7.

45. Ikonomou L, Bastin G, Schneider YJ, Agathos SN. Design of an efficient medium for insect cell growth and recombinant protein production. Vitro Cell Dev Biol Anim. 2001;37(9):549-59.

46. Jarvis DL, Summers MD, Garcia A Jr, Bohlmeyer DA. Influence of different signal peptides and prosequences on expression and secretion of human tissue plasminogen activator in the baculovirus system. J Biol Chem. 1993;268(22):16754-62.

47. van Oers MM, Thomas AA, Moormann RJ, Vlak JM. Secretory pathway limits the enhanced expression of classical swine fever virus E2 glycoprotein in insect cells. J Biotech. 2001;86(1):31-8.

48. Yoon C, Johnston SC, Tang J, Stahl M, Tobin JF, Somers WS. Charged residues dominate a unique interlocking topography in the heterodimeric cytokine interleukin-12. EMBO J. 2000;19(14):3530-41.

49. Luo J, Wu SJ, Lacy ER, Orlovsky Y, Baker A, Teplyakov A, Obmolova G, Heavner GA, Richter HT, Benson J. Structural basis for the dual recognition of IL-12 and IL-23 by ustekinumab. J Mol Biol. 2010;402(5):797-812. 